\title{
Some Liouville theorems and applications
}

\author{
YanYan $\mathrm{Li}^{*}$ \\ Department of Mathematics \\ Rutgers University \\ 110 Frelinghuysen Road \\ Piscataway, NJ 08854 \\ USA \\ Dedicated to Haim Brezis with high respect and friendship
}

2000 Mathematics Subject Classification: 35J60, 35J70, 53A30

\begin{abstract}
We give exposition of a Liouville theorem established in [6] which is a novel extension of the classical Liouville theorem for harmonic functions. To illustrate some ideas of the proof of the Liouville theorem, we present a new proof of the classical Liouville theorem for harmonic functions. Applications of the Liouville theorem, as well as that of earlier ones in [5], can be found in $[6,7]$ and [9].
\end{abstract}

The Laplacian operator $\Delta$ is invariant under rigid motions: For any function $\mathrm{u}$ on $\mathbb{R}^{n}$ and for any rigid motion $T: \mathbb{R}^{n} \rightarrow \mathbb{R}^{n}$,

$$
\Delta(u \circ T)=(\Delta u) \circ T \text {. }
$$

*Partially supported by NSF grant DMS-0401118. To appear in a volume in honor of Haim Brezis' sixtieth birthday. 
The following theorem is classical:

$$
u \in C^{2}, \Delta u=0 \text { and } u>0 \text { in } \mathbb{R}^{n} \text { imply that } u \equiv \text { constant. }
$$

In this note we present a Liouville theorem in [6] which is a fully nonlinear version of the classical Liouville theorem (1).

Let $u$ be a positive function in $\mathbb{R}^{n}$, and let $\psi: \mathbb{R}^{n} \cup\{\infty\} \rightarrow \mathbb{R}^{n} \cup\{\infty\}$ be a Möbius transformation, i.e. a transformation generated by translations, multiplications by nonzero constants and the inversion $x \rightarrow x /|x|^{2}$. Set

$$
u_{\psi}:=\left|J_{\psi}\right|^{\frac{n-2}{2 n}}(u \circ \psi)
$$

where $J_{\psi}$ is the Jacobian of $\psi$.

It is proved in [3] that an operator $H\left(u, \nabla u, \nabla^{2} u\right)$ is conformally invariant, i.e.

$$
H\left(u_{\psi}, \nabla u_{\psi}, \nabla^{2} u_{\psi}\right) \equiv H\left(u, \nabla u, \nabla^{2} u\right) \circ \psi \text { holds for all positive } u \text { and all Möbius } \psi,
$$

if and only if $H$ is of the form

$$
H\left(u, \nabla u, \nabla^{2} u\right) \equiv f\left(\lambda\left(A^{u}\right)\right)
$$

where

$$
A^{u}:=-\frac{2}{n-2} u^{-\frac{n+2}{n-2}} \nabla^{2} u+\frac{2 n}{(n-2)^{2}} u^{-\frac{2 n}{n-2}} \nabla u \otimes \nabla u-\frac{2}{(n-2)^{2}} u^{-\frac{2 n}{n-2}}|\nabla u|^{2} I,
$$

$I$ is the $n \times n$ identity matrix, $\lambda\left(A^{u}\right)=\left(\lambda_{1}\left(A^{u}\right), \cdots, \lambda_{n}\left(A^{u}\right)\right)$ denotes the eigenvalues of $A^{u}$, and $f$ is a function which is symmetric in $\lambda=\left(\lambda_{1}, \cdots, \lambda_{n}\right)$.

Due to the above characterizing conformal invariance property, $A^{u}$ has been called in the literature the conformal Hessian of $u$. Since

$$
\sum_{i=1}^{n} \lambda_{i}\left(A^{u}\right)=-\frac{2}{n-2} u^{-\frac{n+2}{n-2}} \Delta u
$$

Liouville theorem (1) is equivalent to

$$
u \in C^{2}, \quad \lambda\left(A^{u}\right) \in \partial \Gamma_{1} \text { and } u>0 \text { in } \mathbb{R}^{n} \text { imply that } u \equiv \text { constant }
$$

where

$$
\Gamma_{1}:=\left\{\lambda \mid \sum_{i=1}^{n} \lambda_{i}>0\right\} .
$$


Let

$\Gamma \subset \mathbb{R}^{n}$ be an open convex symmetric cone with vertex at the origin

satisfying

$$
\Gamma_{n}:=\left\{\lambda \mid \lambda_{i}>0,1 \leq i \leq n\right\} \subset \Gamma \subset \Gamma_{1} .
$$

Examples of such $\Gamma$ include those given by elementary symmetric functions. For $1 \leq k \leq n$, let

$$
\sigma_{k}(\lambda):=\sum_{1 \leq i_{1}<\cdots<i_{k} \leq n} \lambda_{i_{1}} \cdots \lambda_{i_{k}}
$$

be the $k$-th elementary symmetric function and let $\Gamma_{k}:=\left\{\lambda \in \mathbb{R}^{n} \mid \sigma_{1}(\lambda), \cdots, \sigma_{k}(\lambda)>0\right\}$, which is equal to the connected component of $\left\{\lambda \in \mathbb{R}^{n} \mid \sigma_{k}(\lambda)>0\right\}$ containing the positive cone $\Gamma_{n}$, satisfies (3) and (4).

For an open subset $\Omega$ of $\mathbb{R}^{n}$, consider

$$
\lambda\left(A^{u}\right) \in \partial \Gamma, \quad \text { in } \Omega .
$$

The following definition of viscosity super and sub solutions of (5) has been given in [6].

Definition 1 A positive continuous function $u$ in $\Omega$ is a viscosity subsolution [resp. supersolution] of (5) when the following holds: if $x_{0} \in \Omega, \psi \in C^{2}(\Omega),(u-\psi)\left(x_{0}\right)=0$ and $u-\psi \leq 0$ near $x_{0}$ then

$$
\lambda\left(A^{\psi}\left(x_{0}\right)\right) \in \mathbb{R}^{n} \backslash \Gamma .
$$

[resp. if $(u-\psi)\left(x_{0}\right)=0$ and $u-\psi \geq 0$ near $x_{0}$ then $\left.\lambda\left(A^{\psi}\left(x_{0}\right)\right) \in \bar{\Gamma}\right]$.

We say that $u$ is a viscosity solution of (5) if it is both a viscosity supersolution and a viscosity subsolution.

Remark 1 If a positive $u$ in $C^{1,1}(\Omega)$ satisfies $\lambda\left(A^{u}\right) \in \partial \Gamma$ a.e. in $\Omega$, then it is a viscosity solution of (5).

Here is the Liouville theorem.

Theorem 1 ([6]) For $n \geq 3$, let $\Gamma$ satisfy (3) and (4), and let $u$ be a positive locally Lipschitz viscosity solution of

$$
\lambda\left(A^{u}\right) \in \partial \Gamma \quad \text { in } \mathbb{R}^{n}
$$

Then $u \equiv u(0)$ in $\mathbb{R}^{n}$. 
Remark 2 It was proved by Chang, Gursky and Yang in [1] that positive $C^{1,1}\left(\mathbb{R}^{4}\right)$ solutions to $\lambda\left(A^{u}\right) \in \partial \Gamma_{2}$ are constants. Aobing Li proved in [2] that positive $C^{1,1}\left(\mathbb{R}^{3}\right)$ solutions to $\lambda\left(A^{u}\right) \in \partial \Gamma_{2}$ are constants, and, for all $k$ and $n$, positive $C^{3}\left(\mathbb{R}^{n}\right)$ solutions to $\lambda\left(A^{u}\right) \in \partial \Gamma_{k}$ are constants. The latter result for $C^{3}\left(\mathbb{R}^{n}\right)$ solutions is independently established by Sheng, Trudinger and Wang in [8]. Our proof is completely different.

Remark 3 Writing $u=w^{-\frac{n-2}{2}}$, then

$$
A^{u} \equiv A_{w}:=w \nabla^{2} w-\frac{1}{2}|\nabla w|^{2} I .
$$

Theorem 1, with $\lambda\left(A^{u}\right) \in \partial \Gamma$ being replaced by $\lambda\left(A_{w}\right) \in \partial \Gamma$, holds for $n=2$ as well. See $[6]$.

In order to illustrate some of the ideas of our proof of Theorem 1 in [6], we give a new proof of the classical Liouville theorem (1). We will derive (1) by using the

Comparison Principle for $\Delta$ : Let $\Omega$ be a bounded open subset of $\mathbb{R}^{n}$ containing the origin 0 . Assume that $u \in C_{l o c}^{2}(\bar{\Omega} \backslash\{0\})$ and $v \in C^{2}(\bar{\Omega})$ satisfy

$$
\Delta u \leq 0 \text { in } \Omega \backslash\{0\} \quad \text { and } \quad \Delta v \geq 0 \text { in } \Omega,
$$

and

$$
u>v \quad \text { on } \partial \Omega \text {. }
$$

Then

$$
\inf _{\Omega \backslash\{0\}}(u-v)>0 .
$$

It is easy to see from this proof of the Liouville theorem (1) that the following Comparison Principle for locally Lipschitz viscosity solutions of (5), established in [5, 6], is sufficient for a proof of Theorem 1.

Proposition 1 Let $\Omega$ be a bounded open subset of $\mathbb{R}^{n}$ containing the origin 0 , and let $u \in C_{\text {loc }}^{0,1}(\bar{\Omega} \backslash\{0\})$ and $v \in C^{0,1}(\bar{\Omega})$. Assume that $u$ and $v$ are respectively positive viscosity supersolution and subsolution of (5), and

$$
u>v>0 \quad \text { on } \partial \Omega .
$$

Then

$$
\inf _{\Omega \backslash\{0\}}(u-v)>0 .
$$


For the proof of Proposition 1 and Theorem 1, see [5, 6]. In this note, we give the Proof of Liouville theorem (1) based on the Comparison Principle for $\Delta$. Let

$$
v(x):=\frac{1}{2}\left[\min _{|y|=1} u(y)\right]|x|^{2-n}, \quad v_{1}(x):=\frac{1}{|x|^{n-2}} v\left(\frac{x}{|x|^{2}}\right), \quad u_{1}(x):=\frac{1}{|x|^{n-2}} u\left(\frac{x}{|x|^{2}}\right) .
$$

Since $u_{1}$ and $v_{1}$ are still harmonic functions, an application of the Comparison Principle for $\Delta$ on $\Omega:=$ the unit ball yields

$$
\liminf _{|y| \rightarrow \infty}|y|^{n-2} u(y)>0 .
$$

Lemma 1 For every $x \in \mathbb{R}^{n}$, there exists $\lambda_{0}(x)>0$ such that

$$
u_{x, \lambda}(y):=\frac{\lambda^{n-2}}{|y-x|^{n-2}} u\left(x+\frac{\lambda^{2}(y-x)}{|y-x|^{2}}\right) \leq u(y) \quad \forall 0<\lambda<\lambda_{0}(x),|y-x| \geq \lambda .
$$

Proof. Without loss of generality we may take $x=0$, and we use $u_{\lambda}$ to denote $u_{0, \lambda}$. By the positivity and the Lipschitz regularity of $u$, there exists $r_{0}>0$ such that

$$
r^{\frac{n-2}{2}} u(r, \theta)<s^{\frac{n-2}{2}} u(s, \theta), \quad \forall 0<r<s<r_{0}, \theta \in \mathbb{S}^{n-1} .
$$

The above is equivalent to

$$
u_{\lambda}(y)<u(y), \quad 0<\lambda<|y|<r_{0} .
$$

We know from (7) that, for some constant $c>0$,

$$
u(y) \geq c|y|^{2-n}, \quad|y| \geq r_{0} .
$$

Let

$$
\lambda_{0}:=\left(\frac{c}{\max _{|z| \leq r_{0}} u(z)}\right)^{\frac{1}{n-2}}
$$

Then

$$
u_{\lambda}(y) \leq\left(\frac{\lambda_{0}}{|y|}\right)^{n-2}\left(\max _{|z| \leq r_{0}} u(z)\right) \leq c|y|^{2-n} \leq u(y), \quad \forall 0<\lambda<\lambda_{0},|y| \geq r_{0} .
$$

It follows from (8) and (9) that

$$
u_{\lambda}(y) \leq u(y), \quad \forall 0<\lambda<\lambda_{0},|y| \geq \lambda
$$

Lemma 1 is established.

Because of Lemma 1, we may define, for any $x \in \mathbb{R}^{n}$ and any $0<\delta<1$, that

$$
\bar{\lambda}_{\delta}(x):=\sup \left\{\mu>0\left|u_{x, \lambda}(y) \leq(1+\delta) u(y), \forall 0<\lambda<\mu,\right| y-x \mid \geq \lambda\right\} \in(0, \infty] .
$$


Lemma 2 For any $x \in \mathbb{R}^{n}$ and any $0<\delta<1, \bar{\lambda}_{\delta}(x)=\infty$.

Proof. We prove it by contradiction. Suppose the contrary, then, for some $x \in \mathbb{R}^{n}$ and some $0<\delta<1, \bar{\lambda}_{\delta}(x)<\infty$. We may assume, without loss of generality, that $x=0$, and we use $u_{\lambda}$ and $\bar{\lambda}_{\delta}$ to denote respectively $u_{0, \lambda}$ and $\bar{\lambda}_{\delta}(0)$. Since the harmonicity is invariant under conformal transformations and multiplication by constants, and since

$$
u(y)=u_{\bar{\lambda}_{\delta}}(y)<(1+\delta) u_{\bar{\lambda}_{\delta}}(y), \quad \forall|y|=\bar{\lambda}_{\delta},
$$

an application of $(7)$ yields, using the fact that $\left(u_{\lambda}\right)_{\lambda} \equiv u$,

$$
\inf _{0<|y|<\bar{\lambda}_{\delta}}\left[(1+\delta) u_{\bar{\lambda}_{\delta}}(y)-u(y)\right]>0
$$

Namely, for some constant $c>0$,

$$
(1+\delta) u(y)-u_{\bar{\lambda}_{\delta}}(y) \geq c|y|^{2-n}, \quad \forall|y| \geq \bar{\lambda}_{\delta}
$$

By the uniform continuity of $u$ on the ball $\left\{z|| z \mid<\bar{\lambda}_{\delta}\right\}$, there exists $0<\epsilon<\bar{\lambda}_{\delta}$ such that for all $\bar{\lambda}_{\delta} \leq \lambda \leq \bar{\lambda}_{\delta}+\epsilon$, and for all $|y| \geq \lambda$, we have

$$
\begin{aligned}
(1+\delta) u(y)-u_{\lambda}(y) & \geq(1+\delta) u(y)-u_{\bar{\lambda}_{\delta}}(y)+\left[u_{\bar{\lambda}_{\delta}}(y)-u_{\lambda}(y)\right] \\
& \geq c|y|^{2-n}-|y|^{2-n}\left|\lambda^{n-2} u\left(\frac{\lambda^{2} y}{|y|^{2}}\right)-\bar{\lambda}_{\delta}^{n-2} u\left(\frac{\bar{\lambda}_{\delta}^{2} y}{|y|^{2}}\right)\right| \geq \frac{c}{2}|y|^{2-n} .
\end{aligned}
$$

This violates the definition of $\bar{\lambda}_{\delta}$. Lemma 2 is established.

By Lemma $2, \bar{\lambda}_{\delta} \equiv \infty$ for all $0<\delta<1$. Namely,

$$
(1+\delta) u(y) \geq u_{x, \lambda}(y), \quad \forall 0<\delta<1, x \in \mathbb{R}^{n},|y-x| \geq \lambda>0 .
$$

Sending $\delta$ to 0 in the above leads to

$$
u(y) \geq u_{x, \lambda}(y), \quad \forall x \in \mathbb{R}^{n},|y-x| \geq \lambda>0 .
$$

This easily implies $u \equiv u(0)$. Liouville theorem (1) is established. 


\section{References}

[1] S.Y.A. Chang, M. Gursky and P. Yang, A prior estimate for a class of nonlinear equations on 4-manifolds, Journal D'Analyse Journal Mathematique 87 (2002), 151186.

[2] A. Li, Liouville type theorem for some degenerate conformally invariant fully nonlinear equation, in preparation.

[3] A. Li and Y.Y. Li, On some conformally invariant fully nonlinear equations, Comm. Pure Appl. Math. 56 (2003), 1416-1464.

[4] A. Li and Y.Y. Li, On some conformally invariant fully nonlinear equations, Part II: Liouville, Harnack and Yamabe, Acta Math. 195 (2005), 117-154.

[5] Y.Y. Li, Degenerate conformally invariant fully nonlinear elliptic equations, arXiv:math.AP/0504598 v1 29 Apr 2005; v2 24 May 2005; final version, to appear in Arch. Rational Mech. Anal.

[6] Y.Y. Li, Local gradient estimates of solutions to some conformally invariant fully nonlinear equations, arXiv:math.AP/0605559 v1 20 May; v2 7 Jul 2006.

[7] Y.Y. Li, Local gradient estimates of solutions to some conformally invariant fully nonlinear equations, C. R. Math. Acad. Sci. Paris, Ser. I, to appear.

[8] W. Sheng, N.S. Trudinger and X.J. Wang, The Yamabe problem for higher order curvatures, arXiv:math.DG/0505463 v1 23 May 2005.

[9] X.J. Wang, Apriori estimates and existence for a class of fully nonlinear elliptic equations in conformal geometry, Chin. Ann. Math., to appear. 